\title{
Accurate Characterization of Dynamic Cell Load in Noise-Limited Random Cellular Networks
}

\author{
Gourab Ghatak $^{\dagger} \ddagger$, Antonio De Domenico ${ }^{\dagger}$, and Marceau Coupechoux ${ }^{\ddagger}$ \\ ${ }^{\dagger}$ CEA, LETI, MINATEC, F-38054 Grenoble, France; ${ }^{\ddagger}$ LTCI, Telecom ParisTech, Université Paris Saclay, France. \\ Email: gourab.ghatak@cea.fr; antonio.de-domenico@cea.fr, and marceau.coupechoux@telecom-paristech.fr
}

\begin{abstract}
DThe analyses of cellular network performance based on stochastic geometry generally ignore the traffic dynamics in the network. This restricts the proper evaluation and dimensioning of the network from the perspective of a mobile operator. To address the effect of dynamic traffic, recently, the mean cell approach has been introduced, which approximates the average network load by the zero cell load. However, this is not a realistic characterization of the network load, since a zero cell is statistically larger than a random cell drawn from the population of cells, i.e., a typical cell. In this paper, we analyze the load of a noise-limited network characterized by high signal to noise ratio (SNR). The noise-limited assumption can be applied to a variety of scenarios, e.g., millimeter wave networks with efficient interference management mechanisms. First, we provide an analytical framework to obtain the cumulative density function of the load of the typical cell. Then, we obtain two approximations of the average load of the typical cell. We show that our study provides a more realistic characterization of the average load of the network as compared to the mean cell approach. Moreover, the prescribed closed-form approximation is more tractable than the mean cell approach.
\end{abstract}

\section{INTRODUCTION}

Stochastic geometry has emerged as an important tool for modeling and analyzing large scale wireless cellular networks [1], wherein the performance is typically characterized by studying metrics such as signal to interference plus noise ratio (SINR) coverage probability and user throughput. To effectively model the user throughput and to efficiently dimension a cellular network from the operators' perspective, the characterization of the cell load is necessary. The existing literature in stochastic geometry models the cell load by considering the average number of associated full buffer users, uniformly distributed over the cell area, see e.g., [1], [2]. This is not realistic since it ignores the effect of dynamic traffic on the user distribution: users with low data rate tend to stay longer in the system, and as a result, the user distribution becomes inhomogeneous in space.

However, studying dynamic traffic using elements of queuing theory in stochastic geometry based analyses is still an open problem [1]. In this regard, Blaszczyszyn et al. [3], have introduced the mean cell approach which avoids extensive simulations by approximating the spatial SINR distribution of a cell with the SINR distribution of the typical user. Thus, in essence, the mean cell approach characterizes the load of the cell containing the typical user, i.e., the zero cell [4]. Although this approach enables modeling the cell load, it may lead to intractable expressions consisting of multiple

The research leading to these results are jointly funded by the European Commission (EC) H2020 and the Ministry of Internal affairs and Communications (MIC) in Japan under grant agreements $\mathrm{N}^{o} 723171$ 5G MiEdge. integrals for evaluation of the SINR coverage probability. Moreover, a characterization of the load of the zero cell is not a reliable metric for evaluating the network wide load distribution since the zero cell is statistically larger than a random cell drawn from the population of cells, i.e., a typical cell. To understand this intuitively, one can assume a random sample point and select the cell containing the point. By stationarity, the distribution of this cell coincides with that of the zero cell. Since the sample point tends to fall with greater probability into larger cells, the zero cell tends to be larger than the typical cell. In this paper, for the case of noise-limited networks, we provide approximations for the network load by characterizing the load of the typical cell. This provides a more realistic characterization of the network load. This noiselimited assumption can be applied to a variety of contexts. For example, in millimeter wave (mm-wave) networks that utilize directional antennas and advanced interference management mechanisms, the performance tends to be noise-limited. Singh et.al [5] have shown the validity of the noise-limited network assumption in mm-wave mesh networks. Furthermore, this noise-limited scenario enables us to visualize our results in light of the seminal work of Bonald et al. [6] who derived the cell load expressions for a single cell with dynamic traffic.

The contribution of this paper is as follows. We obtain a closed-form expression for the cumulative density function (CDF) of the load of the typical cell in a noise-limited network by considering dynamic traffic. We use it to obtain the fraction of stable cells for a given deployment density of small cells. Then, we obtain a single integral-based approximation, and a closed-form expression, for the average load of the typical cell. We show that the first approximation models the cell load from a network perspective more accurately than the mean cell approach. Whereas, the closed-form expression provides a faster and more tractable alternative to calculate the network load, since it does not require evaluation of integrals.

The rest of the paper is organized as follows. In Section [II we introduce the single tier network and the associated parameters. In Section III we present our main results on the CDF and the average of the load of the typical cell of the network. In Section IV we present the results on the stable fraction of the network and we show the accuracy of our derived approximations with respect to Monte-Carlo simulations. Finally, the paper concludes in Section $\mathrm{V}$

\section{SYSTEM MODEL}

We consider a single-tier cellular network equipped with advanced interference management algorithms, so that the user performance is noise-limited. 
The positions of the base stations (BS) are modeled as points of a Poisson point process (PPP) $\phi$ with intensity $\lambda\left[\mathrm{m}^{-2}\right]$. The BSs operate with a transmit power $P_{t}$, and the product of the gains of the antennas at the transmitter and the receiver is $G_{0}$. We consider a fast fading that is Rayleigh distributed with variance equal to one. Furthermore, we assume a path loss model where the power at the origin received from a BS located at a distance $r$ is given by $P_{r}=K \cdot P_{t} \cdot h \cdot G_{0} \cdot r^{-\alpha}$, where $K$ is the path loss coefficient, $h$ is the exponentially distributed fading power, and $\alpha$ is the path loss exponent. Thus, the average SNR can be written as $\frac{K \cdot P_{t} \cdot G_{0} \cdot r^{-\alpha}}{N_{0} \cdot B}=\xi r^{-\alpha}$, where $\xi=\frac{K \cdot P_{t} \cdot G_{0}}{N_{0} \cdot B}$ is the average SNR at $1 \mathrm{~m} . N_{0}$ and $B$ are the noise power density and the operating bandwidth, respectively.

In this network, we assume that the users arrive in the system, download a file, and leave the system. Any new download by the same user is considered as a new user. The arrival process of the new users is Poisson distributed with an intensity $\lambda_{U}$ [users $\cdot \mathrm{s}^{-1} \cdot \mathrm{m}^{-2}$ ] and these new users are uniformly distributed over the network area $A$. The average file size is $\sigma$ [bits/user]. When there are $n$ users simultaneously served by a BS, the available resources are equally shared between them in a Round Robin fashion. Accordingly, we define the traffic density $w$ in the network as $w=\lambda_{U} \cdot \sigma$ $\left[\right.$ bits $\left.\cdot \mathrm{s}^{-1} \cdot \mathrm{m}^{-2}\right]$. Note that, while the user arrivals are uniform in space, as the space-time process evolves, users farther from the serving BSs, i.e., characterized by lower data rates, stay longer in the system, resulting in an inhomogeneous distribution of active users in the network.

\section{Characterization of the Network LoAD}

\section{A. Static vs Dynamic Load}

Before proceeding to our main results, it is necessary to discuss the distinction of dynamic cell-load characterization as compared to the approaches that model the cell load as simply the average number of associated users to a BS [7]. For this we compare the downlink user throughput using the two approaches to study the difference. First, in our system model, we calculate the dynamic cell load using the study of Bonald et.al. [6], say $\bar{\rho}$. Then use it to calculate the downlink user throughput $R_{d y n}$, which is given by [6]:

$$
R_{d y n}=w \frac{1-\bar{\rho}}{\bar{\rho}} \cdot A,
$$

where, the area of the typical cell $A$ is approximated as $A=\frac{1}{\lambda}$, and the average number of active users in the dynamic traffic model [3] is: $N=\frac{\bar{\rho}}{1-\bar{\rho}}$. To compare $R_{d y n}$ with that obtained using the analyses in the existing literature [2], [7], we assume that the users are located homogeneously in each cell of the network following a PPP with an average of $N$ users per cell. Using this assumption, we carry out simulations to obtain the downlink user throughput $T_{P P P}$ as:

$$
R_{P P P}=\mathbb{E}_{n}\left[\frac{B}{n} \log _{2}(1+S I N R)\right],
$$

where the expectation is taken with respect to the number of users in each cell $(n)$, which is Poisson distributed with mean $N$, as well as the SINR of each user in each cell. The difference between $R_{d y n}$ and $R_{P P P}$ is highlighted in Fig. 1 .

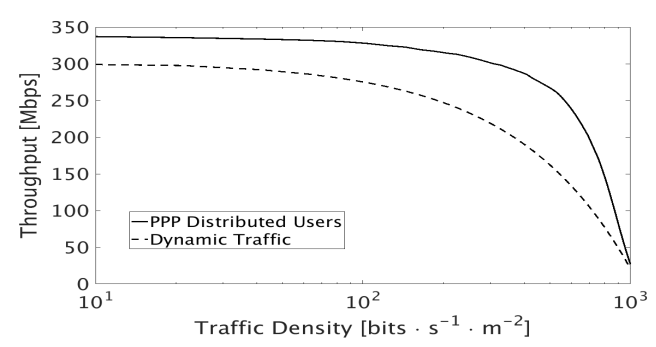

Figure 1: Throughput comparison of mean cell approach with PPP distributed users.

Even though the average number of users in both cases are same, as the space-time process with dynamic traffic evolves, the user distribution is no longer homogeneous in space which is not taken into account in existing studies.

\section{B. Preliminaries}

In case of single-tier random cellular networks, the cell of a BS is given by the Poisson-Voronoi (PV) partition of the space [4]. In the $\mathbb{R}^{2}$ plane, the PV region of a BS located at $x_{0} \in \phi$ is: $\mathcal{A}=\left\{y:\left\|y-x_{0}\right\|<\left\|y-x_{i}\right\| ; \forall x_{i} \in \phi \backslash\left\{x_{0}\right\}\right\}$.

The mosaic of the cells formed for all such $x_{0}$ from a PPP is called a PV network. To investigate the geometry-dependent characteristics of the cells (e.g., the cell load), in a stationary random PV network, it is imperative to define the notion of the 'average' cell. Thus, we recall the following definitions that provide a characterization of the average cell.

Definition 1. The zero cell or the Crofton cell of the PV network is defined as the cell containing a given fixed point in its interior [4].

Definition 2. The typical cell of a PV network is defined as a cell selected at random within a large region of the network with equal chances for each cell to be picked [4]. Thereafter, the network is translated so that the center of the typical cell becomes the origin.

The zero cell versus typical-cell approaches of modeling the network performance corresponds to the evaluation from the perspectives of the user and the network operator, respectively. Hence, for the case of analyzing the network load, the zero cell perspective is not an accurate way of characterization. In what follows, we first define the load of the typical-cell and then, discuss how the load of the zero cell is generally obtained. Then we present our analysis to characterize the load of the typical cell and hence the average network load.

1) Average Load of the Typical Cell: The load of the typical cell in the network can be calculated as:

$$
\rho=\int_{\mathcal{A}} \frac{w}{C(s)} d s
$$

where $C(s)$ is the rate that a user located at $s$ receives in the typical cell $\mathcal{A}$, calculated using the Shannon formula. The random variable $\rho$ characterizes the load of the cell centered at $x_{0}$, and depends on the shape and size of $\mathcal{A}$.

The average load of the typical cell is then calculated by taking the expected values of loads for different realizations of the PPP itself: $\bar{\rho}=\mathbb{E}[\rho]$, 
2) Mean Cell Approximation: In case of PV cells, the average load of the typical cell is generally difficult to evaluate because the shape and size of the typical cells is random. However, by using the ergodicity of the PPP, the area of the typical cell can be approximated as $\frac{1}{\lambda}$ [7]. Then, by assuming the network to be noise-limited, the average load can be approximated using the mean cell approach [3], as:

$$
\bar{\rho}_{M C}=\int_{T} \frac{w}{B \lambda \log _{2}(1+T)} p(T) d T,
$$

where the expectation is taken with respect to the signal to noise ratio (SNR) $(T)$ variations averaged over the fast fading, and $p(T)=\frac{-d \mathbb{P}_{C}(T)}{d T}$ is the probability density function (pdf) of the SNR of the typical user obtained by differentiating the SNR coverage probability, $\mathbb{P}_{C}(T)$. Thus, the cell load can be calculated numerically, given the SNR distribution.

However in the mean cell approach, as the expectation is taken with respect to the SNR variations of the typical user, it calculates the expected load of the zero cell which is statistically larger than the typical cell [4]. Thus, the mean cell approach, always overestimates the load of the typical cell. In the next sections, we propose a new approximation, which is both more accurate and more tractable. First, we derive the CDF of the cell load of the typical cell using the distribution of its area. Then, we obtain a single-integral-based and a closedform approximation for the average load of the typical cell.

\section{Distribution of the Area of the Typical Cell}

The reduced area of a PV cell is defined as [4] :

$$
s=A / \mathbb{E}[A],
$$

where $A$ is the area of the typical cell, and $\mathbb{E}[\cdot]$ is the expectation operator. The pdf of the reduced area of the typical PV cell in two dimensions is given by [8]:

$$
f_{s}(x)=\frac{343}{15} \sqrt{\frac{7}{2 \pi}} x^{5 / 2} \exp \left(-\frac{7}{2} x\right) .
$$

Using this, we can obtain the CDF of the area as given below.

Lemma 1. The CDF of the area of the typical $P V$ cell for a $P P P$ with intensity $\lambda$ is given by:

$$
F_{A}(x)=\frac{343}{15} \sqrt{\frac{7}{2 \pi}}\left(\frac{2}{7}\right)^{7 / 2} \gamma_{i n c}\left(\frac{7 \lambda x}{2}, \frac{7}{2}\right),
$$

where $\gamma_{\text {inc }}(\cdot)$ is the lower incomplete gamma function given by: $\gamma_{i n c}(x, a)=\int_{0}^{x} t^{a-1} \exp (-t) d t$.

Proof. The CDF can be easily derived using the relation (5) and integrating (6).

\section{Distribution of the Load of the Typical Cell}

For obtaining the CDF of the load of the typical cell, we assume that the shape of PV cells is circular. Although in a real PV network, almost surely no circular cells occur, our results show that the circular assumption does not greatly deteriorate the derived approximation. Accordingly, the load of a typical cell (3) with area $\mathrm{A}$ is approximated as [6]:

$$
\rho_{T C}(A) \approx \rho_{A P}(A)=\int_{0}^{2 \pi} \int_{0}^{\sqrt{\frac{A}{\pi}}} \frac{w r}{B \lambda \log _{2}\left(1+\xi r^{-2}\right)} d r d \theta .
$$

Theorem 1. The distribution of the load of the typical cell, $\rho$ is given by:

$$
F_{\rho}(l)=F_{A}\left(\pi\left(\frac{1}{\xi} \exp \left(-\frac{\alpha}{2} E i^{-1}\left(-\frac{l}{K^{\prime}}\right)\right)\right)^{\frac{-2}{\alpha}}\right)
$$

where $K^{\prime}=\frac{4 w \pi \ln (2) \xi}{\alpha^{2} B \lambda} \xi^{\frac{2}{\alpha}}$ and the symbol $E i^{-1}(x)$ is the inverse of the exponential integral. For the special case of $\alpha=2$, it is approximated as:

$$
F_{\rho}(l) \approx F_{A}\left(\frac{\xi \pi\left(1+\exp \left(-\frac{l}{K_{1}}\right)\right)}{\exp \left(-\frac{l}{K_{1}}\right)}\right),
$$

where $K_{1}=\frac{w \pi \ln (2) \xi}{B \lambda}$.

Proof. According to our assumption of high SNR for dense networks, we can approximate $1+\xi r^{-\alpha}$ as $\xi r^{-\alpha}$. Substituting $\ln \left(\xi r^{-\alpha}\right)=y$, we have:

$$
\begin{aligned}
\rho_{A P}(A) & =K^{\prime} \int_{\ln \left(\xi(\pi / A)^{\frac{\alpha}{2}}\right.}^{\infty} \frac{\exp (-y)}{y} d y \\
& =K^{\prime} \mathrm{E}_{1}\left(\frac{2}{\alpha} \ln \left(\xi\left(\frac{\pi}{A}\right)^{\frac{\alpha}{2}}\right)\right)
\end{aligned}
$$

where, $K^{\prime}=\frac{4 w \pi \ln (2) \xi}{\alpha^{2} B \lambda} \xi^{\frac{2}{\alpha}}$ and $\mathrm{E}_{1}(\cdot)$ is the exponential integral function [9]. The $\mathrm{CDF}$ is then simply obtained by some algebraic manipulations of the expression $\mathbb{P}\left(\rho_{A P}(A) \leq l\right)$. For the special case of $\alpha=2$, the CDF of the approximated load of the typical cell $\rho_{A P}(A)$ is derived as:

$$
\mathbb{P}\left(\rho_{A P}(A) \leq l\right)=\mathbb{P}\left(-\ln \left(\xi \pi A^{-1}\right) \leq \mathrm{Ei}^{-1}\left(-\frac{l}{K_{1}}\right)\right),
$$

where, the symbol $\mathrm{Ei}^{-1}(x)$ is given by $\operatorname{Ei}(x)=-\mathrm{E}_{1}(-x)$. Although an explicit expression for $\mathrm{Ei}^{-1}($.$) does not exist, Pecina$ [10] provided piece-wise functions to approximate $\mathrm{Ei}^{-1}(x)$ for different ranges of $x$. The asymptotic approximation for $\frac{-1}{K_{1}} \rightarrow 0$ is [10]:

$$
\mathrm{Ei}^{-1}\left(-\frac{l}{K_{1}}\right) \approx \frac{\exp \left(-\frac{l}{K_{1}}\right)}{1+\exp \left(-\frac{l}{K_{1}}\right)} .
$$

In our analysis, we assume $P_{t}=30 \mathrm{dBm}$, a noise density of $-174 \mathrm{dBm} / \mathrm{Hz}$, and $B=1 \mathrm{GHz}$. The path loss coefficient $K$ is derived from the Umi model for data transmission [11]. As the load $l$ varies from $0 \leq l \leq 1$, for $G_{0}=20 \mathrm{~dB}$, and $\lambda=1 e-5 \mathrm{~m}^{-2}$, we have $0 \leq \frac{l}{K_{1}} \leq 1 e-8$. Thus, our asymptotic approximation is valid. Using this approximation completes the proof.

\section{E. Proposed Approximations for the Average Load of the} Typical Cell

Using the distribution of (7) and 10 we obtain the following approximation of the average load:

$$
\bar{\rho}_{A P}=\int_{0}^{\infty} K^{\prime} \mathrm{E}_{1}\left(\frac{2}{\alpha} \ln \left(\xi\left(\frac{\pi}{A}\right)^{\frac{\alpha}{2}}\right)\right) f_{A}(A) d A,
$$

where $f_{A}(x)=\frac{d F_{A}(x)}{d x}$. Since solving 11) is tedious, we provide two results to approximate the average load of the 


$$
\begin{aligned}
\bar{\rho}_{A P}^{\prime} & =\int_{0}^{\infty} \lambda \frac{2 \pi \ln (2) \xi}{B} F_{1}(A) \frac{343}{15} \sqrt{\frac{7}{2 \pi}}(A \lambda)^{5 / 2} \exp \left(-\frac{7}{2} A \lambda\right) d A \\
\text { where, } & F_{1}(A)= \begin{cases}\frac{\exp \left(-\ln \left(\frac{\xi \pi}{A}\right)\right) \ln \left(\frac{G_{0}}{\ln \left(\frac{\xi \pi}{A}\right)}+G_{0}+\left(1-G_{0}\right) \beta\left(\ln \left(\frac{\xi \pi}{A}\right)\right)\right)}{G_{0}+\left(1-G_{0}\right) \exp \left(\frac{-\ln \left(\frac{\xi \pi}{A}\right)}{1-G_{0}}\right)} ; & A \leq \frac{\pi \xi}{\exp (1)} \\
-\gamma-\ln \left(-\ln \left(\frac{\xi \pi}{A}\right)\right)+\left(-\ln \left(\frac{\xi \pi}{A}\right)\right)-\frac{\left(-\ln \left(\frac{\xi \pi}{A}\right)\right)^{2}}{8} ; & A>\frac{\pi \xi}{\exp (1)}\end{cases}
\end{aligned}
$$

typical cell for the special case of $\alpha=21$. In Section IV we will highlight the advantage of each approximation.

1) Exponential Integral (EI) based Approximation:

Theorem 2. The average load of the typical cell can be approximated as (12).

Proof. We rely on an approximation of the exponential integral provided by Barry et. al. [9]:

$$
\mathrm{E}_{1}(x)=\frac{\exp (-x) \ln \left(\frac{K_{2}}{x}+K_{2}+\left(1-K_{2}\right) \beta(x)\right)}{K_{2}+\left(1-K_{2}\right) \exp \left(\frac{-x}{1-K_{2}}\right)},
$$

where, $K_{2}=\exp (-\gamma)=0.56, \beta(x)=1-\frac{1}{(h(x)+b x)^{2}}$,

$$
h(x)=\frac{1}{1+x \sqrt{x}}+\frac{0.46 x^{\sqrt{\frac{31}{26}}}}{1+0.43 x^{\sqrt{\frac{31}{26}}}}, b \approx 1.04207,
$$

and $\gamma$ is the Euler's constant. This interpolated version of the exponential integral provides a good approximation for $1 \leq$ $x \leq 50$. This corresponds to the range

$$
\frac{\pi \xi}{\exp (50)} \leq A \leq \frac{\pi \xi}{\exp (1)}
$$

For the region of $A$ greater than this range, we use the asymptotic expansion of $E_{1}(x)$ as:

$$
E_{1}(x)=-\gamma-\ln (x)+x-\frac{x^{2}}{8}+\ldots
$$

For practical ranges of cell sizes, the lower bound in (14) always holds (e.g., with $G_{0}=0 \mathrm{~dB}$, the lower bound on the area is $A \geq 2 e-8)$. Now, substituting (10) in (11) and using (13) and (15) to evaluate the integral, completes the proof.

2) closed-form (CF) Approximation: Substituting $\ln \left(\frac{\xi \pi}{A}\right)=t$, the approximated average load of the typical cell 111 becomes:

$$
\bar{\rho}_{A P}=\chi_{2} \int_{-\infty}^{\infty} E_{1}(t) \exp \left(-\frac{7}{2} t\right) F_{2}(t) d t,
$$

where $\chi_{2}=\frac{w \pi \ln (2) \xi}{\lambda B}(\xi \pi \lambda)^{3.5}$ and $F_{2}(t)=$ $\exp \left(-\frac{7 \lambda \xi \pi}{2} \exp (-t)\right)$.

Now, a closed-form solution to this integral does not exist. However, in what follows, we derive an approximate closedform solution for the average load of the typical cell, which we show to be very accurate in Section IV.

\footnotetext{
${ }^{1}$ For dense deployments, the serving BS is generally in line of sight (LOS) which has a path-loss exponent close to 2 for sub- $6 \mathrm{GHz}$ [12] and $\mathrm{mm}$ wave [11] transmissions.
}

Theorem 3. The average load of the typical cell is approximated by the closed-form expression:

$$
\bar{\rho}_{A P}^{\prime \prime}=\chi_{2}\left(I_{1}\left(t_{2}\right)-I_{1}\left(t_{1}\right)+\left(y_{1}-1\right) I_{2}\left(t_{2}\right)-y_{1} I_{2}\left(t_{1}\right)\right)
$$

where,

$I_{1}(x)=\left(\frac{2}{7}\right)^{2}\left(E_{1}(4.5 x)-(1+3.5 x) e^{-3.5 x} E_{1}(x)+\right.$ $\left.\left(\frac{7}{9}\right) \exp (-4.5 x)\right) I_{2}(x)=\frac{2}{7}\left[E_{1}(4.5 x)-e^{-3.5 x} E_{1}(x)\right]$, and, $t_{1}=-\ln \left(-\frac{2}{7 \lambda \xi \pi} \ln (0.1)\right), t_{2}=-\ln \left(-\frac{2}{7 \lambda \xi \pi} \ln (0.9)\right)$ and, $y_{1}=\frac{0.9 t_{1}-0.1 t_{2}}{t_{1}-t_{2}}$.

Proof. We can approximate $F_{2}(t)$, with a piece-wise defined ramp and step function as follows:

$$
\tilde{F}_{2}(t)= \begin{cases}0 ; & t \leq t_{1}, \\ \frac{0.8 t}{t_{2}-t_{1}}+y_{1} ; & t_{1}<t \leq t_{2}, \\ 1 ; & t>t_{2}\end{cases}
$$

where $t_{1}$ and $t_{2}$ are the points corresponding to 10 and 90 percentile values of $F_{2}(t)$, and $y_{1}$ is the intercept. In the next section, we will show that this approximation provides accurate results for the average cell load. With the approximation of (17) in 16 , we have:

$$
\begin{aligned}
\bar{\rho}_{A P}=\chi_{2}\left(\int_{t_{1}}^{t_{2}} E_{1}(x) e^{3.5 x}\right. & \left(\frac{0.8}{t_{2}-t_{1}} x+y_{1}\right) d x+ \\
& \left.\int_{t_{2}}^{\infty} E_{1}(x) e^{-3.5 x} d x\right)
\end{aligned}
$$

Geller and $\mathrm{Ng}$ [13] provided closed-form expressions for both of the above integral types, which we employ to obtain the closed-form for the average cell load.

\section{Simulation Results}

\section{A. CDF of the Load and Stable Fraction of the Network}

To validate the approximation of the CDF of the cell load, we compute the stable fraction of the network, which is defined as the fraction of non-overloaded cells. Mathematically, this is the probability that the load of the typical cell is less than 1. In Fig. 2 we compare the stable fraction of the network for a file size of $\sigma=100 \mathrm{Mb}$, and a user arrival rate of $\lambda_{U}=100 \mathrm{~km}^{-2}$, obtained with the approximation of the CDF derived in Theorem 1 and the one computed from Monte-Carlo simulations of the PPP. This provides dimensioning rules for the operator in terms of the minimum deployment density of $\mathrm{BSs}$ required to achieve a given stable fraction. For example, with a directive antenna gain of $G_{0}=20 \mathrm{~dB}$ and for a load target of 0.5 , the operator must deploy at least $50 \mathrm{BSs}$ $\mathrm{km}^{-2}$. We also observe that the closed-form CDF provides a good approximation of the numerical values, specially for a larger antenna gain $\left(G_{0}=20 \mathrm{~dB}\right)$. Accordingly, the circular 


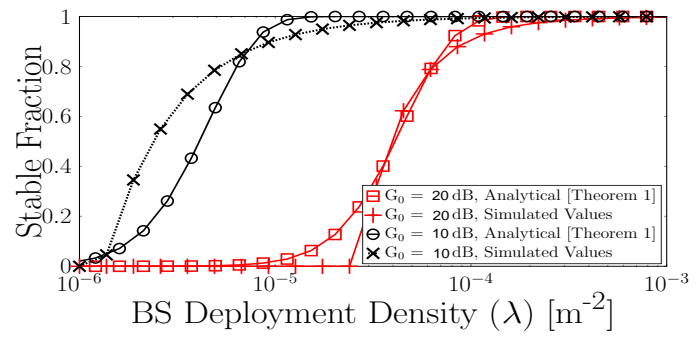

Figure 2: Stable fraction of the network.

assumption of the cell shape is not detrimental for evaluating the performance of the network.

\section{B. Accuracy of the EI Approximation of the Network Load}

In Fig. 3, we compare the average load of the typical cell, computed with the EI approximation (Theorem 2p, the CF expression (Theorem 3), and that obtained using the mean cell approach with the network load calculated using Monte-Carlo simulations. For the Monte-Carlo simulations, we find the average cell load in one realization of the PPP $\phi$, for a given $\lambda, \lambda_{U}$, and $\sigma$ and then we perform the same calculations and average over 1000 PPP realizations. As seen in the figure, the EI approximation provides a more accurate characterization of the network load than the mean cell approach. The mean cell approach always overestimates the actual load, because, the zero cell is, on average, larger than the typical cell, resulting in higher load. Therefore, from the perspective of an operator, we provide a more realistic, and hence reliable method to characterize the network load and to dimension the network. As an example, for $\sigma=100 \mathrm{Mb}$, and $\lambda_{U}=0.01$ users per second, the EI approximation accurately estimates that the operator must deploy $10 \mathrm{BS}$ less (120 as compared to 130) than that prescribed by the mean cell approach.

\section{Advantages of the CF Approximation of the Network Load}

As we see in Fig. 3, the CF approximation provides the loosest approximation to the network load; however, it does not require numerical evaluation of integrals. Moreover, we see that for higher file sizes $(\sigma=100 \mathrm{Mb})$ and denser deployment of small cells $\left(\lambda \geq 1 e-4 \mathrm{~m}^{-2}\right)$, even the CF approximation provides an excellent approximation of the network load.

From a practical perspective, it provides a fast method of accurately estimating the network load without the need of running extensive simulations, which can become infeasible. Particularly, recall that BS locations are Poisson distributed. For every realization of BS locations, SNR distribution should be computed by drawing all required random variables. Moreover, a dynamic traffic of users arriving in the system, downloading a file, and leaving should be simulated for a sufficient duration to reach the mixing time of the Markov process. This procedure should be repeated for every set of possible parameters if we want to characterize the network load. At last cell overloading may be undetectable as any simulation has a finite duration. For all these reasons, the analytical model presented in this analysis is necessary to provide very quick results and interesting insights to the system.

\section{CONClusion}

The realistic assessment of the mobile network performance need to take dynamic traffic into account in order to char-

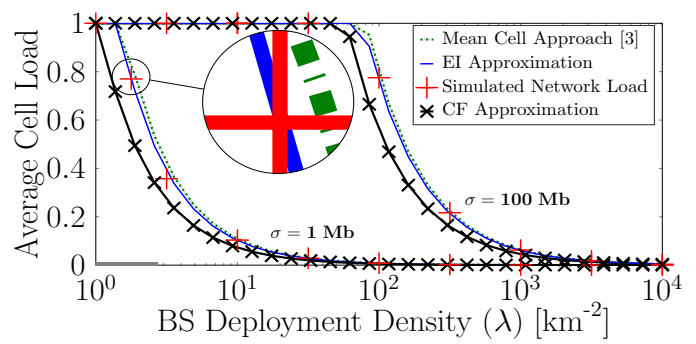

Figure 3: Analytical approximation accuracy, $G_{0}=36 \mathrm{~dB}$.

acterize the network load, which is still an open problem. Towards this end, we have derived a simple approximation for the CDF of the cell load of the typical cell in a noiselimited network, which is characterized by high SINR and low inter-cell interference. Furthermore, we have obtained a single approximation-based expression and a closed-form expression for the average load of the network by using the distribution of the area of the typical cell. Our derivations present a more realistic characterization of the cell load, as compared to the recently introduced mean cell approach, since we consider the typical cell of the network rather than the zero cell. The analysis provides a tractable and accurate characterization for the cell load that can be utilized, e.g., for evaluating the user throughput and dimensioning $5 \mathrm{G}$ networks. However, accurate characterization of the dynamic network load in case of an interference prone network is not straightforward. This will be addressed in a future work.

\section{REFERENCES}

[1] H. ElSawy, E. Hossain, and M. Haenggi, "Stochastic Geometry for Modeling, Analysis, and Design of Multi-Tier and Cognitive Cellular Wireless Networks: A Survey," IEEE Commun. Surveys Tuts., vol. 15, no. 3, pp. 996-1019, 2013.

[2] H. Elshaer, et al., "Downlink and Uplink Cell Association With Traditional Macrocells and Millimeter Wave Small Cells," IEEE Trans. Wireless Commun., vol. 15, no. 9, pp. 6244-6258, Sept 2016.

[3] B. Błaszczyszyn, M. Jovanovicy, and M. K. Karray, "How user throughput depends on the traffic demand in large cellular networks," in IEEE WiOpt, 2014, pp. 611-619.

[4] S. N. Chiu, et al., Stochastic geometry and its applications. John Wiley \& Sons, 2013.

[5] S. Singh, R. Mudumbai, and U. Madhow, "Interference analysis for highly directional 60 -ghz mesh networks: The case for rethinking medium access control," IEEE/ACM Transactions on Networking, vol. 19 , no. 5, pp. 1513-1527, Oct 2011.

[6] T. Bonald and A. Proutière, "Wireless Downlink Data Channels: User Performance and Cell Dimensioning," in ACM MobiCom, 2003.

[7] S. Singh and J. G. Andrews, "Joint Resource Partitioning and Offloading in Heterogeneous Cellular Networks," IEEE Trans. Wireless Commun., vol. 13, no. 2, pp. 888-901, 2014.

[8] M. Tanemura, "Statistical distributions of Poisson Voronoi cells in two and three dimensions," FORMA-TOKYO-, vol. 18, no. 4, 2003.

[9] D. Barry, J.-Y. Parlange, and L. Li, “Approximation for the exponential integral (theis well function)," Journal of Hydrology, vol. 227, no. 1, pp. 287-291, 2000.

[10] P. Pecina, "On the function inverse to the exponential integral function," $B A I C z$, vol. 37, pp. 8-12, 1986.

[11] 3GPP TSG RAN, "TR 38.900, Study on channel model for frequency spectrum above $6 \mathrm{GHz}$, , v14.1.0, September 2016.

[12] —, "TR 36.814, E-UTRA; Further advancements for E-UTRA physical layer aspects," v9.0.0, March 2010.

[13] M. Geller and E. W. Ng, "A table of integrals of the exponential integral," J. Res. Natl. Bur. Stand., vol. 71, pp. 1-20, 1969. 